\title{
Investigating the visual span in comparative search: the effects of task difficulty and divided attention
}

\author{
Marc Pomplun*, Eyal M. Reingold, Jiye Shen \\ Department of Psychology, University of Toronto, 100 St. George Street, Toronto, \\ Ontario, M5S 3G3, Canada
}

Received 2 December 2000; accepted 16 February 2001

\begin{abstract}
In three experiments, participants' visual span was measured in a comparative visual search task in which they had to detect a local match or mismatch between two displays presented side by side. Experiment 1 manipulated the difficulty of the comparative visual search task by contrasting a mismatch detection task with a substantially more difficult match detection task. In Experiment 2, participants were tested in a single-task condition involving only the visual task and a dual-task condition in which they concurrently performed an auditory task. Finally, in Experiment 3, participants performed two dual-task conditions, which differed in the difficulty of the concurrent auditory task. Both the comparative search task difficulty (Experiment 1) and the divided attention manipulation (Experiments 2 and 3) produced strong effects on visual span size. (C) 2001 Elsevier Science B.V. All rights reserved.
\end{abstract}

Keywords: Visual span in comparative search; Task difficulty; Divided attention

\section{Introduction}

The region of the visual field from which we can extract information during an eye fixation has been referred to as the visual span, ${ }^{1}$ the perceptual span, or the span

\footnotetext{
* Corresponding author. Tel.: +1-416-978-3990.

E-mail address: marc@psych.utoronto.ca (M. Pomplun).

${ }^{1}$ In the present paper, the term visual span has been used as synonymous to the terms perceptual span or the span of effective vision to refer to the region from which information was processed during fixations that were produced while participants were performing the comparative visual search task.
}

0010-0277/01/\$ - see front matter (C) 2001 Elsevier Science B.V. All rights reserved. PII: S0010-0277(01)00123-8 
of effective vision (Jacobs, 1986; Rayner, 1998). The measurement of visual span size has been very influential in reading research (e.g. McConkie \& Rayner, 1975; Rayner \& Bertera, 1979) and has recently been applied to visual search (Bertera \& Rayner, 2000; Rayner \& Fisher, 1987a,b; Reingold, Charness, Pomplun, \& Stampe, 2001; see Rayner, 1998, for a review). Most studies employed the gaze-contingent moving window technique to measure visual span. Essentially, this technique obscures all objects from view, except those within a certain 'window' that is continually centered on the participant's current gaze position. The window position changes across fixations to follow the gaze position. For example, in a study by McConkie and Rayner (1975), participants read text that was masked outside a visual window that included the fixated character and a number of characters to the left and to the right. Only the text within the window was legible. The visual span in reading was assessed by varying the window size across trials and determining the smallest window size that allowed participants to read with normal speed.

In the present study, we examined visual span in a comparative visual search task (see Pomplun, 1998; Pomplun et al., in press), in which participants attempted to localize a single mismatch between otherwise identical display halves (Fig. 1a). Visual span was measured by employing the gaze-contingent moving window paradigm (see Fig. 1b,c). An iterative algorithm varied the size of the window over successive trials to efficiently determine the smallest window that did not significantly interfere with the participant's search speed. This enabled a reliable measurement of the participant's visual span with a minimum of experimental trials.

The main goal of the present study was to investigate the effects of task difficulty and divided attention on visual span in the comparative visual search task. In a previous study, Rayner and Fisher (1987a) found that task difficulty influenced visual span in a letter search task in which participants searched through rows of letter strings for a designated target letter. By applying the gaze-contingent moving window technique, Rayner and Fisher showed that participants' visual span decreased with increasing similarity between distractor and target letters. In the current study, Experiment 1 manipulated the difficulty of the comparative visual search task by contrasting a mismatch detection task, as described above, with a match detection task. In the match detection task, corresponding items across display halves did not match in color or shape, except for the target item, which had either the same color or the same shape as its counterpart in the other display half. Response time measurements in previous research on comparative search tasks (Pomplun, 1998) indicated that the mismatch detection task can be performed more efficiently than the match detection task. According to the results obtained by Rayner and Fisher, we would thus expect a smaller visual span for the match detection task than for the mismatch detection task.

In addition to task difficulty, many studies have demonstrated that another factor influencing visual span is foveal load (e.g. Crundall, Underwood, \& Chapman, 1999; Ikeda \& Takeuchi, 1975; Mackworth, 1965; Williams, 1985, 1989; see Williams, 1988, for a review). Such studies typically employed two concurrent visual tasks, one of them involving the presentation of stimuli in the center of the display, the other involving the presentation of stimuli in the periphery. Williams (1989), for 
example, had participants perform a central letter discrimination task that either induced a high or low foveal load. In a simultaneous peripheral task, participants named a one-digit number shown at variable eccentricities. It was found that a more
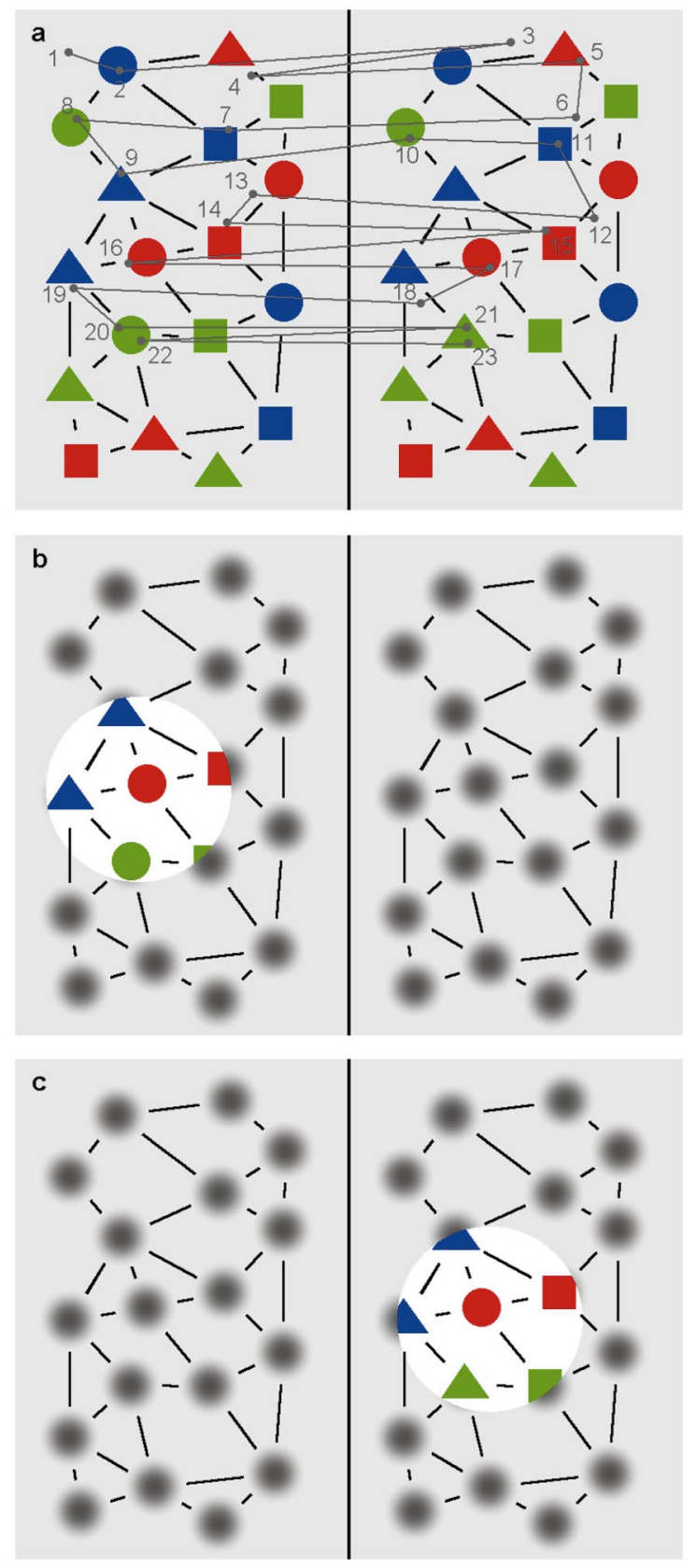
difficult central task (i.e. higher foveal load) decreased participants' performance in the peripheral detection task. In some of these studies (e.g. Mackworth, 1965; Williams, 1985, 1989), investigators found this decline in performance to be greater for targets at larger eccentricities, arguing that the foveal load reduced the useful field of view (i.e. the visual span). They referred to this effect using the controversial term 'tunnel vision' (see Williams, 1988).

Given that foveal load was operationalized using what basically amounts to a dual-task manipulation, it is possible that the reduction in visual span resulted at least in part from the effects of divided attention (for a review of divided attention literature, see Duncan, 1980; Hirst, 1986). That is, the peripheral task and the central processing task may share limited capacity resources resulting in performance decrements. To examine the possible effects of divided attention on visual span under conditions that exclude foveal load as a potential factor, we employed an auditory rather than a visual secondary task. Accordingly, Experiment 2 measured visual span in both a single-task condition (i.e. comparative visual search only) and a dual-task condition (i.e. comparative visual search and a concurrent auditory task). A finding of a reduction in visual span due to the concurrent auditory task would not be attributable to foveal load or low-level perceptual effects such as visual masking (see Breitmeyer, 1984). Finally, Experiment 3 investigated the effects of increasing the difficulty of the secondary auditory task (i.e. easy vs. difficult) on the visual span in the comparative search task.

\section{Method}

\subsection{Participants}

Twenty-four paid participants took part in the present study (eight in each experiment). All participants had normal or corrected-to-normal vision.

\subsection{Materials}

In the comparative visual search task, each half of the display contained 18 objects, which were randomly distributed over a rectangular area of 9.1 visual degrees horizontally and 17.4 degrees vertically with a separation of 3.7 degrees

Fig. 1. Illustration of the comparative search task. Note that object sizes and colors seen here do not correspond exactly to those used in the actual experimental displays. (a) Unmasked stimulus with a participant's visual scan path superimposed upon it (eye fixations are numbered and connected by straight lines). The participants' task was to detect a mismatch between the display halves in either the color or form of one of the objects. Here, a green circle in the lower left half turns into a green triangle in the right half. (b) The same display, with a gaze-contingent window. The window was centered on participants' gaze position and moved following a change in gaze position. Note that unlike in the displays used in the experiment, in the figure the background has been made brighter within the window for illustrative purposes. (c) The same display after the participant switched to the corresponding location in the right half, possibly detecting the mismatch. 
between the halves (see Fig. 1). The objects had diameters of approximately 0.7 degrees and a minimum distance of 2.6 degrees between their centers. Their colors (red, green, and blue) were adjusted to be equiluminant, and their forms (square, circle, and triangle) were adjusted to have the same perceived size. Each object in the left half had a counterpart at the corresponding position in the right half. In mismatch detection trials, each object matched its counterpart in both color and form, except for one target object that was mismatched in either color or form. In match detection trials (used only in Experiment 1), no object matched its counterpart in color or form, except for the target object that was matched in either color or form. Each possible color-form combination occurred exactly twice in each display half, except for one feature in either half being changed to create the target object. Neighboring objects were connected by black lines to facilitate precise eye movements when switching between the display halves. In the gaze-contingent window trials, the objects outside a circular, gaze-centered window were replaced with gray blobs masking the actual colors and forms.

\subsection{Apparatus}

Eye movements were measured with an SR Research Ltd. EyeLink system. Following calibration, gaze-position error was less than 0.5 degrees. The temporal resolution of the system was $4 \mathrm{~ms}$. The gaze-contingent window followed the participant's gaze position with an average delay of $14 \mathrm{~ms}$.

\subsection{Speed measurement}

Participants were asked to systematically scan the display from top to bottom. If they reached the bottom of the display without detecting the target object, they were free to choose any search strategy. These instructions made it possible to accurately assess the participants' vertical search speed (in degrees per second), which was calculated in terms of the time and vertical distance required for the gaze to move from its starting position at the top of the screen to an imaginary horizontal line 1 degree above the target. Trials were discarded and replaced if the participants' gaze position did not reach the imaginary line or if the covered vertical distance was shorter than 5 degrees. This definition of vertical search speed reduced noise and increased measurement reliability.

\subsection{Procedure}

Prior to every trial, participants were asked to fixate a marker in the upper left corner of the display. Following a button press, a search display was presented. As soon as participants detected the target, they ended the trial by pressing another button. The trial was terminated automatically if no response was made within $60 \mathrm{~s}$. Each experiment included two conditions (Experiment 1: match vs. mismatch; Experiment 2: single vs. dual task; Experiment 3: easy vs. difficult auditory task) and started with 20 practice trials followed by nine baseline trials for each condition. In the baseline trials, all objects were visible in the half of the screen that was fixated 
by the participant. All objects in the other half were masked with gray blobs. The normative speed for each condition was calculated as the first quartile of the baseline speed values. Following the baseline trials, 24 blocks with three speed measurements in each block were presented. Each block consisted of trials in one of the two experimental conditions, and conditions were alternated across blocks. For each block, a speed measurement was computed and used to determine the window size of the next block in the same condition. For the first block in each condition, the window size was set to 8 degrees in diameter. The window size was increased if the median of three successive speed measurements was slower than $98 \%$ of the normative speed and decreased if it was faster than $102 \%$ of the normative speed. The first adjustment in each condition was an increase or decrease of 1.28 degrees, and each successive adjustment was $11 \%$ smaller than the preceding one. Consequently, the final adjustment in the sequence of 12 represented a change of only 0.32 degrees. The visual span size for each condition was calculated as the mean of the last two window sizes (i.e. the window sizes after adjustments 11 and 12). To account for any changes in performance over time due to practice or fatigue, the normative speed was updated by administering four baseline trials after every sequence of three adjustments. These trials replaced the four earliest baseline trials, and the normative speed was recomputed.

Experiment 1 used a 'mismatch detection' and a 'match detection' condition. Experiment 2 contrasted a 'single-task' with a 'dual-task' condition. In every trial, participants performed a mismatch detection task, while a stream of random numbers between 1 and 10 was presented auditorily at a computer-regulated rate of one per second. In the 'single-task' condition, participants were told to ignore these numbers. In the 'dual-task' condition, participants were forewarned that once the trial was over, they would be asked to report how many odd numbers had been presented. This question was displayed on the screen in a four-alternative multiplechoice format after the completion of the trial. Participants answered by pressing one of four buttons and were immediately informed whether their choice was correct.

In Experiment 3, participants performed in 'easy auditory task' and 'difficult auditory task' conditions. Before each trial, a list of target numbers was shown at the center of the screen. In the 'easy auditory task' condition, the list contained only one number - the number 5 - whereas in the 'difficult auditory task' condition, the list contained

four randomly chosen numbers (which varied across trials). Participants had to memorize these numbers without any time limit imposed on them. During the trial, a stream of random numbers between 1 and 10 was presented auditorily at a rate of one per $1.5 \mathrm{~s}$. The frequency of auditory targets was the same for both conditions such that on average, every third number was one of the previously memorized numbers. Participants had to press a button whenever they heard a target number. When participants failed to press the button within $1.5 \mathrm{~s}$, a high-pitch sound was presented.

\section{Results}

In each experiment, an error in the comparative visual search task was registered 
when no response was made within $60 \mathrm{~s}$ (timeout) or when no fixations had occurred closer than 2 degrees to the target within the last second before the trial was terminated by the participant. In general, error rates were very low across all experimental conditions in all of the experiments (Experiment 1: mismatch detection, 1.5\%; match detection, 2.1\%; $t<1$; Experiment 2: single task, 1.4\%; dual task, 1.6\%; $t<1$; Experiment 3: easy auditory task, $1.5 \%$; difficult auditory task, $1.6 \% ; t<1$ ). The error rates in the auditory task were substantial in the dual-task condition in Experiment 2 (44.9\%) and relatively low in Experiment 3 (easy auditory task, 2.2\%; difficult auditory task, $8.5 \% ; t(7)=5.73, P<0.01)$. In the remainder of the results section, performance on correct baseline trials and visual span estimates are reported for each of the three experiments.

In Experiment 1, there were longer reaction times (RTs) and more fixations for match than mismatch detection (RT: mismatch detection, $9.04 \mathrm{~s}$; match detection, $15.46 \mathrm{~s} ; t(7)=2.91, P<0.05$; number of fixations: mismatch detection, 36.9 ; match detection, $58.1 ; t(7)=2.41, P<0.05)$, indicating that mismatch detection was performed more efficiently than match detection. This was also signified by shorter saccades within display halves and longer fixation duration in the match task than in the mismatch task (fixation duration: mismatch detection, $200 \mathrm{~ms}$; match detection, $217 \mathrm{~ms} ; t(7)=3.13, P<0.05$; saccade amplitude: mismatch detection, 3.75 degrees; match detection, 2.79 degrees; $t(7)=2.99, P<0.05$ ). Fig. 2a shows the average window size across participants for each of the 12 adjustments made in Experiment 1. As can clearly be seen, the window size decreased faster for the match condition, asymptoting at a substantially lower level than for the mismatch condition. According to the last two adjustments in each condition, the span diameter was 7.3 and 2.9 degrees for mismatch and match detection, respectively $(t(7)=4.86$, $P<0.01)$. It is important to note that the visual span area in the match condition (6.4 degrees $^{2}$ ) was equivalent to less than a sixth the size of that in the mismatch condition $\left(41.5\right.$ degrees $^{2}$ ). If objects and fixations were to be uniformly distributed across the display, then these span areas would translate to 0.8 objects within the window for the match condition, as opposed to 5.5 objects within the window for the mismatch condition. Although there were large individual differences, every single participant showed a substantial difference in visual span between the two conditions, demonstrating the robustness and reliability of the observed effect.

In Experiment 2, RT was longer and the number of fixations was larger in the dual than in the single task (RT: single task, $8.09 \mathrm{~s}$; dual task, $14.21 \mathrm{~s} ; t(7)=10.20$, $P<0.001$; number of fixations: single task, 32.4 ; dual task, $47.2 ; t(7)=5.82$, $P<0.01$ ), indicating that the simultaneous auditory task reduced performance in the visual task. Fixations were longer in the dual-task than in the single-task condition (single task, $202 \mathrm{~ms}$; dual task, $248 \mathrm{~ms} ; t(7)=5.79, P<0.01$ ), while saccade amplitude within display halves was not significantly influenced by the experimental condition (single task, 2.79 degrees; dual task, 2.80 degrees; $t<1$ ). As shown in Fig. 2 b, there was a substantial difference in visual span size across the two conditions. Across the 12 adjustments, the window size decreased faster for the dual-task condition and stabilized at a clearly lower level than for the single-task condition. The visual span diameters were determined as 7.1 and 4.1 degrees for single and 
a

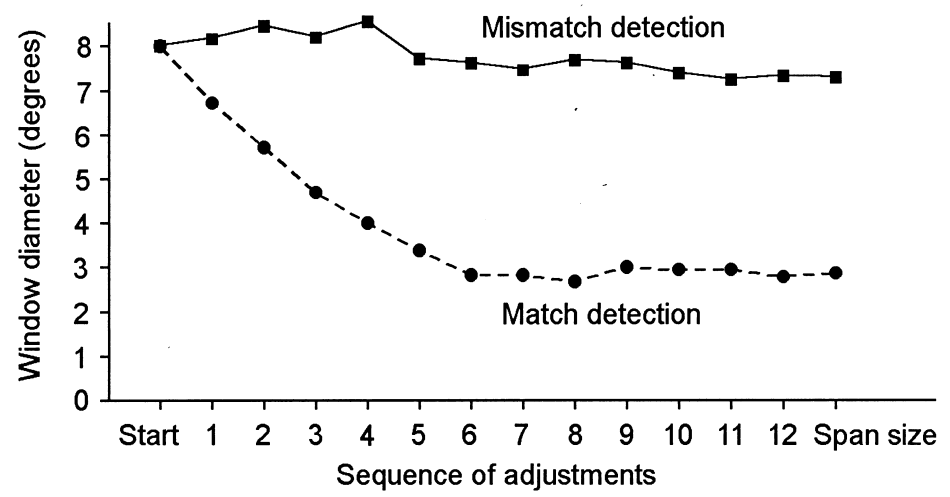

b

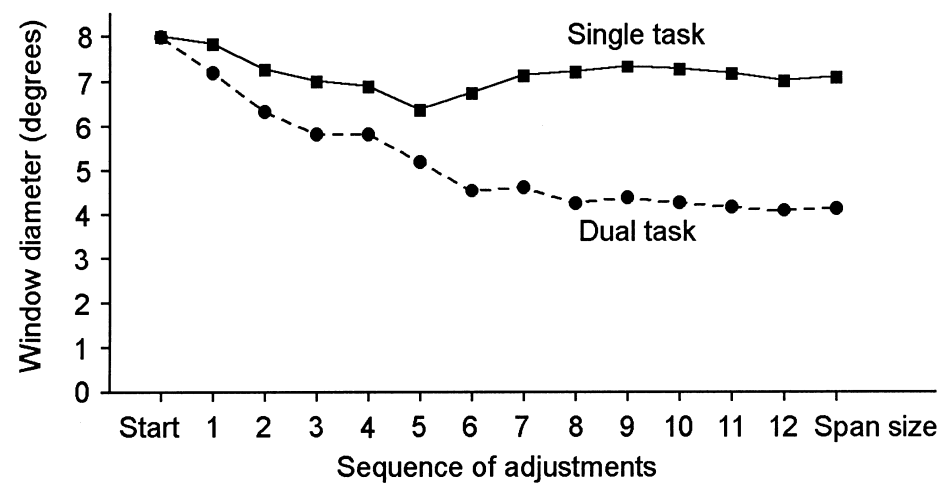

C

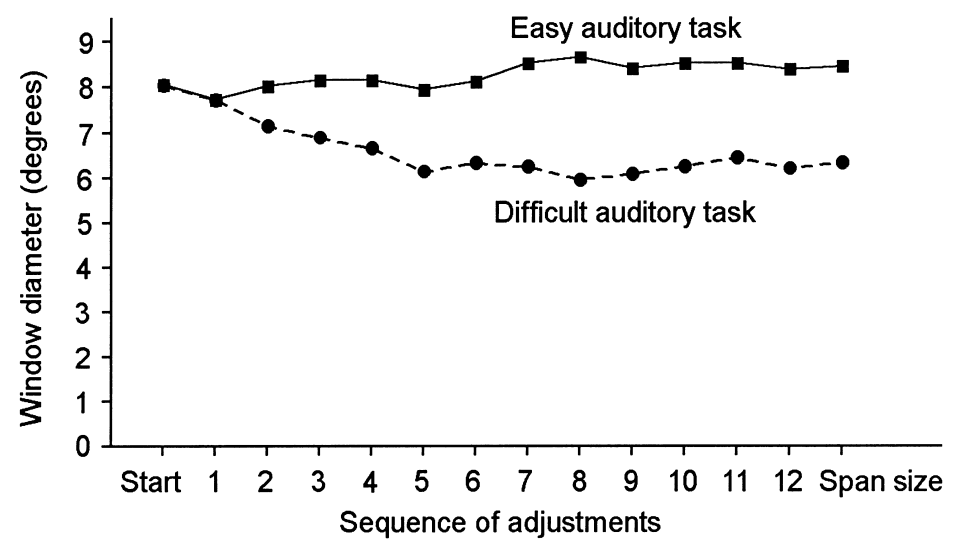

Fig. 2. Mean window sizes in Experiments 1-3 (a-c, respectively) at the beginning of the experiment and after each of the 12 adjustments. The visual span sizes were defined as the mean of the last two values for each condition. 
dual task, respectively $(t(7)=6.25, P<0.001)$. This corresponds to a visual span area of 39.5 degrees $^{2}$ or 5.2 objects in the single-task condition and an area of 13.5 degrees $^{2}$ or 1.8 objects in the dual-task condition. Thus, in the dual-task condition, the concurrent auditory task reduced the visual span area to one-third of its size in the single-task condition.

In Experiment 3, there was a tendency towards longer RTs in the difficult than in the easy auditory task condition, whereas the number of fixations did not differ across conditions (RT: easy task, $6.56 \mathrm{~s}$; difficult task, $8.50 \mathrm{~s} ; t(7)=2.32$, $P=0.054$; number of fixations: easy task, 30.0 ; difficult task, $35.6 ; t(7)=1.63$, $P=0.147)$. Fixations were longer and saccades were shorter in the difficult than in the easy auditory task condition (fixation duration: easy task, $170 \mathrm{~ms}$; difficult task, $189 \mathrm{~ms} ; t(7)=4.46, P<0.01$; saccade amplitude: easy task, 2.92 degrees; difficult task, 2.70 degrees; $t(7)=4.12, P<0.01$ ). While the window size in the easy-task condition did not change substantially across the 12 adjustments, it strongly decreased in the difficult-task condition (see Fig. 2c), resulting in a considerable difference in visual span size (easy task, 8.5 degrees; difficult task, 6.4 degrees; $t(7)=2.97, P<0.05)$. This corresponds to a visual span area of 56.8 degrees $^{2}$ or 7.1 objects within the window for the easy-task condition and an area of 32.2 degrees $^{2}$ or 4.1 objects for the difficult-task condition. In the difficult-task condition, the visual span area was only $57 \%$ of its size in the easy-task condition.

\section{Discussion}

Consistent with the study by Rayner and Fisher (1987a), the present findings document a substantial reduction in visual span associated with increasing task difficulty (Experiment 1). Moreover, by employing a concurrent auditory task (Experiments 2 and 3), the present study demonstrates clear effects of divided attention on visual span, which cannot be attributed to foveal load or low-level visual masking (see Jolicoeur, 1999; Pashler, 1994, for other demonstrations of cross-modal, dual-task interference). This result points out the possibility that previous reports on the effects of foveal load on visual span size (e.g. Crundall et al., 1999; Ikeda \& Takeuchi, 1975; Mackworth, 1965; Williams, 1985, 1988, 1989) may actually reflect at least in part the effects of divided attention.

The present study constitutes another illustration of the usefulness of the gazecontingent moving window paradigm. Specifically, in the present experiments, the combination of the gaze-contingent display technique and the iterative algorithm of window size adjustment provided a direct and efficient method for visual span measurement (see Reingold et al., 2001, for another application of this methodology).

Although it is not always easy to generalize laboratory findings to real-world situations, the present study has direct implications for a variety of applied settings. For example, Ball, Owsley, Sloane, Roenker, and Bruni (1993), in a large-scale driving study, found a negative correlation between visual span in a laboratory task and accident involvement. The present experiments have demonstrated that 
auditory distraction reduces the size of the visual span, and that this reduction increases with more demanding auditory tasks. It is thus hardly surprising that talking on cellular telephones is also correlated with car accidents - regardless of whether the phone is hands-free or not (Redelmeier \& Tibshirani, 1997). The demonstration in Experiment 1 that visual span is also reduced when the difficulty of the visual task increases suggests that auditory distraction may be even more dangerous when traffic or road conditions are impaired. In view of the present results, it seems advisable to avoid demanding auditory tasks during the performance of critical visual tasks.

\section{Acknowledgements}

We thank Alex Gunz for his helpful comments on an earlier version of this manuscript. Preparation of this paper was supported by a grant to E.M.R. from the Natural Science and Engineering Research Council of Canada (NSERC) and a grant to M.P. from the Deutsche Forschungsgemeinschaft (DFG).

\section{References}

Ball, K., Owsley, C., Sloane, M. E., Roenker, D. L., \& Bruni, J. R. (1993). Visual attention problems as a predictor of vehicle crashes in older drivers. Investigative Ophthalmology \& Visual Science, 34, 3110 3123.

Bertera, J. H., \& Rayner, K. (2000). Eye movements and the span of the effective visual stimulus in visual search. Perception \& Psychophysics, 62, 576-585.

Breitmeyer, B. G. (1984). Visual masking: an integrative approach. Oxford: Clarendon.

Crundall, D. E., Underwood, G., \& Chapman, P. R. (1999). Driving experience and the functional field of view. Perception, 28, 1075-1087.

Duncan, J. (1980). The locus of interference in the perception of simultaneous stimuli. Psychological Review, 87, 272-300.

Hirst, W. (1986). Aspects of divided and selective attention. In J. LeDoux, \& W. Hirst (Eds.), Mind and brain (pp. 105-141). New York: Cambridge University Press.

Ikeda, M., \& Takeuchi, T. (1975). Influence of foveal load on the functional visual field. Perception \& Psychophysics, 18, 255-260.

Jacobs, A. M. (1986). Eye movement control in visual search: how direct is visual span control? Perception \& Psychophysics, 39, 47-58.

Jolicoeur, P. (1999). Dual-task interference and visual encoding. Journal of Experimental Psychology: Human Perception and Performance, 25, 596-616.

Mackworth, N. H. (1965). Visual noise causes tunnel vision. Psychonomic Science, 3, 67-68.

McConkie, G. W., \& Rayner, K. (1975). The span of the effective stimulus during a fixation in reading. Perception \& Psychophysics, 17, 578-586.

Pashler, H. (1994). Dual-task interference in simple tasks: data and theory. Psychological Bulletin, 116, 220-244.

Pomplun, M. (1998). Analysis and models of eye movements in comparative visual search. Göttingen: Cuvillier.

Pomplun, M., Sichelschmidt, L., Wagner, K., Clermont, T., Rickheit, G., \& Ritter, H. (2001). Comparative visual search: a difference that makes a difference. Cognitive Science 25(1), 3-36.

Rayner, K. (1998). Eye movements in reading and information processing: 20 years of research. Psychological Bulletin, 124, 372-422.

Rayner, K., \& Bertera, J. H. (1979). Reading without a fovea. Science, 206, 468-469. 
Rayner, K., \& Fisher, D. L. (1987a). Eye movements and the perceptual span during visual search. In J. K. O'Regan, \& A. Levy-Schoen (Eds.), Eye movements: from physiology to cognition (pp. 293-302). Amsterdam: North-Holland.

Rayner, K., \& Fisher, D. L. (1987b). Letter processing during eye fixations in visual search. Perception \& Psychophysics, 42, 87-100.

Redelmeier, D. A., \& Tibshirani, R. J. (1997). Association between cellular-telephone calls and motor vehicle collisions. New England Journal of Medicine, 336, 453-458.

Reingold, E. M., Charness, N., Pomplun, M., \& Stampe, D. M. (2001). Visual span in expert chess players: evidence from eye movements. Psychological Science, 12, 49-56.

Williams, L. J. (1985). Tunnel vision induced by a foveal load manipulation. Human Factors, 27, 221227.

Williams, L. J. (1988). Tunnel vision or general interference? Cognitive load and attentional bias are both important. American Journal of Psychology, 101, 171-191.

Williams, L. J. (1989). Foveal load affects the functional field of view. Human Performance, 2, 1-28. 